International Electronic Journal of Algebra

VOLUME 21 (2017) 55-75

\title{
DERIVATIONS OF TERNARY LIE ALGEBRAS AND GENERALIZATIONS
}

\author{
Amine Ben Abdeljelil, Mohamed Elhamdadi and Abdenacer Makhlouf \\ Received: 18 February 2016 \\ Communicated by Sergei Silvestrov
}

\begin{abstract}
We study derivations of ternary Lie algebras. Precisely, we investigate the relation between derivations of Lie algebras and the induced ternary Lie algebras. We also explore the spaces of quasi-derivations, the centroid and the quasi-centroid and give some properties. Finally, we compute these spaces for low dimensional ternary Lie algebras $\mathfrak{g}$.
\end{abstract}

Mathematics Subject Classification (2010): 20B25, 20 B20

Keywords: Ternary Lie algebra, ternary Lie algebra induced by Lie algebra, derivation, central derivation, generalized derivation

\section{Introduction}

Derivations of different algebraic structures are an important subject of study in algebra and diverse areas. They appear in many fields of mathematics and physics. In particular, they appear in representation theory and cohomology theory among other areas. They have various applications relating algebra to geometry and allow the construction of new algebraic structures. In this context, derivations of Lie algebras [11], Hom-Lie algebras [15] and Lie triple systems [12] have been studied. Indeed, many generalizations were introduced. For instance, generalized derivations, quasi-derivations of Lie algebras [11], $(\alpha, \beta, \gamma)$-derivations [13], $\delta$-derivations [5] have been investigated. See also the following references for further studies $[3,4,6,7,8,9,10,14]$. In the present paper we are interested in studying the derivations of Lie algebras and derivations of induced ternary Lie algebras. In particular, we investigate the relation between derivations of Lie algebras and the induced ternary Lie algebras. We also introduce substructures of the $\operatorname{space} \operatorname{Der}(\mathfrak{g})$ of derivations, study their properties and give some examples.

This paper is organized as follows. In Section 2, we review the basics of ternary Lie algebras, give some examples and recall the construction given in [2] that allows to induce ternary Lie algebras by a Lie algebra and a trace function. Section 3 deals with derivations of ternary Lie algebras and some generalizations. We discuss the 
space $\operatorname{Der}(\mathfrak{g})$ and other subspaces, we give their properties and study the connection between derivations of Lie algebras and induced ternary Lie algebras. We show that if $\mathfrak{g}$ is a ternary Lie algebra with trivial center which can be decomposed to a sum of ideals then we can reduce the study of its derivations to those of the components. Moreover we discuss centroids, quasi-derivations, quasi-centroids, $(\alpha, \beta, \gamma, \theta)$-derivations and $(\alpha, \beta, \gamma, \theta)$-quasiderivations. In Section 4 , we compute the set of derivations and other generalized derivations of low dimensional ternary Lie Algebras.

\section{Ternary Lie algebras}

First we review some definitions needed through the paper and we illustrate them by giving some examples.

Definition 2.1. Let $[\cdot, \cdot, \cdot]$ be a skew-symmetric trilinear map on a $\mathbb{K}$-vector space $\mathfrak{g}$. We say that $(\mathfrak{g},[\cdot, \cdot, \cdot])$ is a ternary Lie algebra or a 3-Lie algebra if the map $[\cdot, \cdot, \cdot]$ satisfies for all $x_{1}, . ., x_{5} \in \mathfrak{g}$ the identity

$\left[x_{1}, x_{2},\left[x_{3}, x_{4}, x_{5}\right]\right]=\left[\left[x_{1}, x_{2}, x_{3}\right], x_{4}, x_{5}\right]+\left[x_{3},\left[x_{1}, x_{2}, x_{4}\right], x_{5}\right]+\left[x_{3}, x_{4},\left[x_{1}, x_{2}, x_{5}\right]\right]$.

The previous identity is called Nambu identity or sometimes fundamental identity or Filippov identity.

Example 2.2. Let $\mathrm{V}$ be a three-dimensional vector space with the basis $\left\{\boldsymbol{e}_{1}, e_{2}, e_{3}\right\}$. Any skew-symmetric trilinear map $[\cdot, \cdot, \cdot]: \mathrm{V} \longrightarrow \mathrm{V}$ satisfies the identity (1). To verify this we let $i, j=1,2,3$ with $i<j$ and by an easy computation we obtain

$$
\left[\left[e_{i}, e_{j}, e_{1}\right], e_{2}, e_{3}\right]+\left[e_{1},\left[e_{i}, e_{j}, e_{2}\right], e_{3}\right]+\left[e_{1}, e_{2},\left[e_{i}, e_{j}, e_{3}\right]\right]=\left[e_{i}, e_{j},\left[e_{1}, e_{2}, e_{3}\right]\right] .
$$

Example 2.3. Let $M_{n}(\mathbb{C})$ be the space of $n \times n$ matrices over the field of complex numbers. The bracket $[A, B, C]=\circlearrowleft \operatorname{Tr}(A) \Gamma(B, C)$, where $\operatorname{Tr}$ is the trace function and $\Gamma$ is the commutator operator defined by $\Gamma(\mathrm{A}, \mathrm{B})=\mathrm{AB}-\mathrm{BA}$, this bracket gives $\mathrm{M}_{\mathrm{n}}(\mathbb{C})$ a ternary Lie algebra structure. The symbol $\circlearrowleft$ means that we are taking a cyclic summation on $\mathrm{A}, \mathrm{B}, \mathrm{C}$.

Example 2.4. The polynomial algebra of 3 variables $x_{1}, x_{2}, x_{3}$, with the bracket defined by the functional jacobian:

$$
\left[f_{1}, f_{2}, f_{3}\right]=\left|\begin{array}{lll}
\frac{\partial f_{1}}{\partial x_{1}} & \frac{\partial f_{1}}{\partial x_{2}} & \frac{\partial f_{1}}{\partial x_{3}} \\
\frac{\partial f_{2}}{\partial x_{1}} & \frac{\partial f_{2}}{\partial x_{2}} & \frac{\partial f_{2}}{\partial x_{3}} \\
\frac{\partial f_{3}}{\partial x_{1}} & \frac{\partial f_{3}}{\partial x_{2}} & \frac{\partial f_{3}}{\partial x_{3}}
\end{array}\right|
$$

is a ternary Lie algebra. 
Example 2.5. The following ternary Lie algebra is the only 4-dimensional simple ternary Lie algebra. The bracket are defined with respect to the basis $\left\{e_{1}, e_{2}, e_{3}, e_{4}\right\}$ by

$$
\begin{aligned}
& {\left[e_{1}, e_{2}, e_{3}\right]=-e_{4},} \\
& {\left[e_{1}, e_{2}, e_{4}\right]=e_{3},} \\
& {\left[e_{1}, e_{3}, e_{4}\right]=-e_{2},} \\
& {\left[e_{2}, e_{3}, e_{4}\right]=e_{1} .}
\end{aligned}
$$

Remark 2.6. The previous Definition 2.1 can obviously be generalized to higher dimension by defining an $\mathrm{n}$-ary Lie bracket with the following generalization of Nambu's identity:

$$
\left[x_{1}, . ., x_{n-1},\left[y_{1}, . ., y_{n}\right]\right]=\sum_{i=1}^{n}\left[y_{1}, . ., y_{i-1},\left[x_{1}, . ., x_{n-1}, y_{i}\right], y_{i+1}, . ., y_{n}\right]
$$

A vector space $\mathfrak{g}$ with a skew-symmetric $\mathrm{n}$-ary bracket satisfying this identity is called an $\mathrm{n}$-ary Lie Algebra or $\mathrm{n}$-Lie Algebra.

Definition 2.7. Let $(\mathfrak{g},[\cdot, \cdot, \cdot])$ be a ternary Lie algebra and let $\mathfrak{h}$ be a subspace of $\mathfrak{g}$.

- We say that $\mathfrak{h}$ is a ternary Lie sub-algebra of $(\mathfrak{g},[\cdot, \cdot, \cdot])$ if it is closed under the bracket, that is if $[\mathfrak{h}, \mathfrak{h}, \mathfrak{h}] \subseteq \mathfrak{h}$.

- A subspace $\mathcal{I}$ of $\mathfrak{g}$ is called an ideal if $[\mathcal{I}, \mathfrak{g}, \mathfrak{g}] \subset \mathcal{I}$.

- A ternary Lie algebra is said to be simple if it has no proper ideal.

- The center of $(\mathfrak{g},[\cdot, \cdot, \cdot])$ is the set

$$
Z(\mathfrak{g})=\left\{\mathfrak{u} \in \mathfrak{g} ;\left[u, x_{1}, x_{2}\right]=0, \text { for all } x_{1}, x_{2} \in \mathfrak{g}\right\} .
$$

$\mathbf{Z}(\mathfrak{g})$ is an abelian ideal of $\mathfrak{g}$.

An easy fact is that the center of a non-abelian simple ternary Lie algebra is trivial.

- The subspace $\mathfrak{g}^{1}=[\mathfrak{g}, \mathfrak{g}, \mathfrak{g}]$ is a ternary Lie sub-algebra of $\mathfrak{g}$ called the derived algebra.

- A morphism of ternary Lie algebras, is a linear map $\varphi:\left(\mathfrak{g},[\cdot, \cdot, \cdot]_{\mathfrak{g}}\right) \longrightarrow$ $\left(\eta,[\cdot, \cdot, \cdot]_{\eta}\right)$ such that for any $x, y, z \in \mathfrak{g}$ we have

$$
\varphi\left([x, y, z]_{\mathfrak{g}}\right)=[\varphi(x), \varphi(y), \varphi(z)]_{\eta} .
$$

Remark 2.8. As in the case of Lie algebras, the kernel of a ternary Lie algebras morphism is an ideal of $\mathfrak{g}$. In fact, if $\mathfrak{u}$ is in $\operatorname{ker}(\varphi)$ then for any $\boldsymbol{v}, \boldsymbol{w} \in \mathfrak{g}$, 
$\varphi\left([u, v, w]_{\mathfrak{g}}\right)=[\varphi(\mathfrak{u}), \varphi(v), \varphi(w)]_{\eta}=0$.

However, its image $\mathfrak{I}(\varphi)$ is not always an ideal but a ternary Lie sub-algebra of $\eta:$ For $v_{1}, v_{2}, v_{3} \in \mathfrak{I}(\varphi)$ we have $\left[v_{1}, v_{2}, v_{3}\right]_{\eta}=\left[\varphi\left(u_{1}\right), \varphi\left(u_{2}\right), \varphi\left(u_{3}\right)\right]_{\eta}=$ $\varphi\left(\left[\mathfrak{u}_{1}, \mathfrak{u}_{2}, \mathfrak{u}_{3}\right]_{\mathfrak{g}}\right)$ for some $\mathfrak{u}_{1}, \mathfrak{u}_{2}, \mathfrak{u}_{3} \in \mathfrak{g}$.

The two following propositions are given in [15] in a context of Hom-Lie algebras, here we state them in the case of ternary Lie algebras.

Proposition 2.9. Given two ternary Lie algebras $(\mathfrak{g},[\cdot, \cdot, \cdot])$ and $\left(\eta,[\cdot, \cdot, \cdot]_{\eta}\right)$, the space $\mathfrak{g} \oplus \eta$ with the bracket defined by

$$
\left[\left(u_{1}, v_{1}\right),\left(u_{2}, v_{2}\right),\left(u_{3}, v_{3}\right)\right]_{\mathfrak{g} \oplus \eta}=\left(\left[u_{1}, u_{2}, u_{3}\right]_{\mathfrak{g}},\left[v_{1}, v_{2}, v_{3}\right]_{\mathfrak{\eta}}\right)
$$

is a ternary Lie algebra.

Proof. Obvious.

Proposition 2.10. A linear map $\varphi:\left(\mathfrak{g},[\cdot, \cdot, \cdot]_{\mathfrak{g}}\right) \longrightarrow\left(\eta,[\cdot, \cdot, \cdot]_{\mathfrak{\eta}}\right)$ is morphism of ternary Lie algebras if and only if its graph $\mathcal{G}_{\varphi}$ is a ternary Lie sub-algebra of $\left(\mathfrak{g} \oplus \eta,[\cdot, \cdot, \cdot]_{\mathfrak{g} \oplus \boldsymbol{\eta}}\right)$.

Proof. Suppose that $\varphi:\left(\mathfrak{g},[\cdot, \cdot, \cdot]_{\mathfrak{g}}\right) \longrightarrow\left(\eta,[,, \cdot, \cdot]_{\eta}\right)$ is morphism of ternary Lie algebras and let $\mathfrak{u}, v, w \in \mathfrak{g}$, we have

$$
\begin{aligned}
{[(u, \varphi(u)),(v, \varphi(v)),(w, \varphi(w))]_{\mathfrak{g} \oplus \mathfrak{\eta}} } & =\left([u, v, w]_{\mathfrak{g}},[\varphi(u), \varphi(v), \varphi(w)]_{\mathfrak{\eta}}\right) \\
& =\left([u, v, w]_{\mathfrak{g}}, \varphi\left([u, v, w]_{\mathfrak{g}}\right)\right) \in \mathcal{G}_{\varphi} .
\end{aligned}
$$

Then $\mathcal{G}_{\varphi}$ is closed under the bracket $[\cdot, \cdot, \cdot]_{\mathfrak{g} \oplus \boldsymbol{\eta}}$.

Conversely, if $\mathcal{G}_{\varphi}$ is a ternary Lie sub-algebra of $\left(\mathfrak{g} \oplus \eta,[\cdot, \cdot, \cdot]_{\mathfrak{g} \oplus \mathfrak{\eta}}\right)$, then

$$
\mathcal{G}_{\varphi} \ni[(u, \varphi(u)),(v, \varphi(v)),(w, \varphi(w))]_{\mathfrak{g} \oplus \eta}=\left([u, v, w]_{\mathfrak{g}},[\varphi(u), \varphi(v), \varphi(w)]_{\eta}\right) .
$$

Thus $[\varphi(u), \varphi(v), \varphi(w)]_{\eta}=\varphi\left([u, v, w]_{\mathfrak{g}}\right)$.

2.1. Ternary Lie algebras induced by Lie algebras. In [2], the authors gave a procedure to construct a ternary Lie algebra structure from a Lie bracket over the same vector space using a trace map. Precisely, we have the following.

Proposition 2.11. [2] Let $(\mathfrak{g},[\cdot, \cdot])$ be a Lie algebra and $\tau: \mathfrak{g} \longrightarrow \mathbb{K}$ be a trace map on $\mathfrak{g}$, then $\left(\mathfrak{g},[\cdot, \cdot, \cdot]_{\mathcal{\tau}}\right)$ is a ternary Lie algebra, where

$$
[x, y, z]_{\tau}=\tau(x)[y, z]+\tau(z)[x, y]+\tau(y)[z, x] .
$$

The ternary Lie algebra $\left(\mathfrak{g},[\cdot, \cdot, \cdot]_{\tau}\right)$ is called the ternary Lie algebra induced by the Lie algebra $(\mathfrak{g},[\cdot, \cdot])$ and the trace map $\tau$. 
Remark 2.12. We recall that a trace function $\tau: \mathfrak{g} \longrightarrow \mathbb{K}$ is a linear map such that $\tau([x, y])=0$ for all $x, y \in \mathfrak{g}$.

We give an example to illustrate this construction.

Example 2.13. Let $\mathrm{H}_{2}$ be the five dimensional Heisenberg Lie algebra with generators $\mathrm{P}_{1}, \mathrm{P}_{2}, \mathrm{Q}_{1}, \mathrm{Q}_{2}$ and $\mathrm{Z}$ subject to the following bracket relations (unspecified bracket relations are given by skew-symmetry or are zeros):

$$
\left[\mathrm{P}_{1}, \mathrm{Q}_{1}\right]=\left[\mathrm{P}_{2}, \mathrm{Q}_{2}\right]=\mathrm{Z} \text {, and }\left[\mathrm{P}_{1}, \mathrm{Q}_{2}\right]=\left[\mathrm{P}_{2}, \mathrm{Q}_{1}\right]=-\mathrm{Z} \text {. }
$$

Since $\mathbf{Z}$ is the only bracket, then any linear map $\tau: \mathrm{H}_{2} \longrightarrow \mathbb{K}$ such that $\tau(\mathbf{Z})=0$, is a trace function on $\mathrm{H}_{2}$. Then $\left(\mathrm{H}_{2},[\cdot, \cdot, \cdot]_{\tau}\right)$ is a ternary Lie algebra with the following ternary brackets:

$$
\begin{aligned}
& {\left[P_{1}, P_{2}, Q_{1}\right]_{\tau}=\tau\left(P_{1}-P_{2}\right) Z,} \\
& {\left[P_{1}, P_{2}, Q_{2}\right]_{\tau}=\tau\left(P_{1}+P_{2}\right) Z,} \\
& {\left[Q_{1}, Q_{2}, P_{1}\right]_{\tau}=\tau\left(Q_{1}+Q_{2}\right) Z,} \\
& {\left[Q_{1}, Q_{2}, P_{2}\right]_{\tau}=\tau\left(Q_{1}-Q_{2}\right) Z .}
\end{aligned}
$$

A converse construction is also possible; if $(\mathfrak{g},[\cdot, \cdot, \cdot])$ is a ternary Lie algebra, we can induce a Lie algebra structure on $\mathfrak{g}$. Fix an element $\omega \in \mathfrak{g}$ and define the bracket $[x, y]_{\omega}=[x, y, \omega]$, then $\left(\mathfrak{g},[\cdot, \cdot]_{\omega}\right)$ is a Lie algebra. In fact $[\cdot, \cdot]_{\omega}$ is clearly bilinear and skew-symmetric and by a direct computation one can see that it satisfies the Jacobi identity.

\section{Derivations of ternary Lie algebras and ternary Lie algebras induced by Lie algebras}

Now let us define derivations of a ternary Lie algebra and some other generalizations.

\subsection{Derivations, central derivations and centroids.}

Definition 3.1. Let $(\mathfrak{g},[\cdot, \cdot, \cdot])$ be a ternary Lie algebra, and D a linear map on $\mathfrak{g}$. $\mathrm{D}$ is said to be a derivation of $\mathfrak{g}$ if

$$
\mathrm{D}\left(\left[\mathrm{x}_{1}, \mathrm{x}_{2}, \mathrm{x}_{3}\right]\right)=\left[\mathrm{D}\left(\mathrm{x}_{1}\right), \mathrm{x}_{2}, \mathrm{x}_{3}\right]+\left[\mathrm{x}_{1}, \mathrm{D}\left(\mathrm{x}_{2}\right), \mathrm{x}_{3}\right]+\left[\mathrm{x}_{1}, \mathrm{x}_{2}, \mathrm{D}\left(\mathrm{x}_{3}\right)\right]
$$

for all $\chi_{1}, \chi_{2}, \chi_{3} \in \mathfrak{g}$. We denote by $\operatorname{Der}(\mathfrak{g})$ the space of derivations of $\mathfrak{g}$. 
Example 3.2. A straightforward computation gives the following fact: If $\mathrm{D}$ is a derivation of the ternary Lie algebra in Example 2.5 with its matrix $M=\left(a_{i j}\right)_{1 \leq i, j \leq 4}$ with respect to the basis $\left\{\mathrm{e}_{1}, \mathrm{e}_{2}, \mathrm{e}_{3}, \mathrm{e}_{4}\right\}$ then

$$
M=\left(\begin{array}{cccc}
0 & a_{12} & a_{13} & a_{14} \\
-a_{12} & 0 & a_{23} & a_{24} \\
-a_{13} & -a_{23} & 0 & a_{34} \\
-a_{14} & -a_{24} & -a_{34} & 0
\end{array}\right)
$$

Remark 3.3. $\operatorname{Der}(\mathfrak{g})$ is a Lie algebra with the bracket

$$
\left[\mathrm{D}_{1}, \mathrm{D}_{2}\right]=\mathrm{D}_{1} \circ \mathrm{D}_{2}-\mathrm{D}_{2} \circ \mathrm{D}_{1} .
$$

$\operatorname{Der}(\mathfrak{g})$ can also be equipped with a ternary Lie algebra structure induced from this Lie bracket:

Proposition 3.4. Let $\mathfrak{g}$ be a finite dimensional Lie algebra. Consider the map $\tau: \operatorname{Der}(\mathfrak{g}) \longrightarrow \mathbb{K}$ defined by $\tau(\mathrm{D})=\operatorname{tr}(\mathrm{D})$ consisting of the trace of the matrix of $\mathrm{D}, \tau$ is a trace function on $\operatorname{Der}(\mathfrak{g})$ and it follows that $\left(\operatorname{Der}(\mathfrak{g}),[\cdot, \cdot, \cdot]_{\tau}\right)$ is a ternary Lie algebra.

Proposition 3.5. Let $(\mathfrak{g},[\cdot, \cdot, \cdot])$ be a ternary Lie algebra with trivial center and which can be decomposed as the direct sum of two ideals: $\mathfrak{g}=\mathcal{I} \oplus \mathcal{J}$, then we have

$$
\operatorname{Der}(\mathfrak{g})=\operatorname{Der}(\mathcal{I}) \oplus \operatorname{Der}(\mathcal{J}) .
$$

To prove this proposition, we are going to use the following lemma:

Lemma 3.6. Let $(\mathfrak{g},[\cdot, \cdot, \cdot])$ be a ternary Lie algebra such that $\mathfrak{g}=\mathcal{I} \oplus \mathcal{J}$, where $\mathcal{I}$ and $\mathcal{J}$ are two ideals of $\mathfrak{g}$. Suppose that $\mathrm{Z}(\mathfrak{g})=\{0\}$, then for any $\mathrm{D} \in \operatorname{Der}(\mathfrak{g})$, we have $\mathrm{D}(\mathcal{I}) \subseteq \mathcal{I}$ and $\mathrm{D}(\mathcal{J}) \subseteq \mathcal{J}$.

Proof. Let $u \in \mathcal{I}$, such that $\mathrm{D}(\mathrm{u})=v_{1}+v_{2}$, where $v_{1} \in \mathcal{I}$ and $v_{2} \in \mathcal{J}$. Let $x, y \in \mathfrak{g}$. We have

$$
\begin{aligned}
{\left[v_{2}, x, y\right] } & =\left[D(u)-v_{1}, x, y\right] \\
& =[D(u), x, y]-\left[v_{1}, x, y\right] \\
& =D([u, x, y])-[u, D(x), y]-[u, x, D(y)]-\left[v_{1}, x, y\right] .
\end{aligned}
$$

Since $\mathcal{I}$ is an ideal of $\mathfrak{g}$, therefore $[u, D(x), y],[u, x, D(y)]$ and $\left[v_{1}, x, y\right]$ are in $\mathcal{I}$.

Now write $x=x_{1}+x_{2}$ and $y=y_{1}+y_{2}$ such that $x_{1}, y_{1} \in \mathcal{I}$ and $x_{2}, y_{2} \in \mathcal{J}$, then

$$
[u, x, y]=\left[u, x_{1}, y_{1}\right]+\left[u, x_{1}, y_{2}\right]+\left[u, x_{2}, y_{1}\right]+\left[u, x_{2}, y_{2}\right] \text {. }
$$

Each of $\left[u, x_{1}, y_{2}\right],\left[u, x_{2}, y_{1}\right],\left[u, x_{2}, y_{2}\right]$ is in $\mathcal{I} \cap \mathcal{J}$, so they are all zeros, thus 


$$
\begin{aligned}
\mathrm{D}([\mathrm{u}, x, y]) & =\mathrm{D}\left(\left[u, x_{1}, y_{1}\right]\right) \\
& =\left[\mathrm{D}(u), x_{1}, y_{1}\right]+\left[u, D\left(x_{1}\right), y_{1}\right]+\left[u, x_{1}, D\left(y_{1}\right)\right] .
\end{aligned}
$$

Then $\mathrm{D}([u, x, y]) \in \mathcal{I}$. It follows that $\left[v_{2}, x, y\right] \in \mathcal{I} \cap \mathcal{J}$, then $\left[v_{2}, x, y\right]=0$. Hence $v_{2}=0$ since $\mathrm{Z}(\mathfrak{g})=0$.

We can now prove Proposition 3.5.

Proof. By the previous lemma, we can see that a restriction of any derivation of $\mathfrak{g}$ to $\mathcal{I}$ (respectively $\mathcal{J}$ ) is a derivation of $\mathcal{I}$ (respectively $\mathcal{J}$ ).

A natural question is about derivations of ternary Lie algebras induced by a Lie bracket and how they can be related to derivations of the original Lie algebra.

Proposition 3.7. Let $(\mathfrak{g},[\cdot, \cdot])$ be a Lie algebra and $\left(\mathfrak{g},[\cdot, \cdot, \cdot]_{\tau}\right)$ be an induced ternary Lie algebra. Let $\mathrm{D}$ be a derivation of $(\mathfrak{g},[\cdot, \cdot])$. If $\mathrm{D}(\mathfrak{g}) \subset \operatorname{Ker}(\tau)$, then $\mathrm{D}$ is a derivation of $\left(\mathfrak{g},[\cdot, \cdot, \cdot]_{\tau}\right)$.

Proof. Let D be a derivation of $(\mathfrak{g},[\cdot, \cdot])$. For all $x, y, z \in \mathfrak{g}$,

$$
\begin{aligned}
{[\mathrm{D}(x), y, z]_{\tau}+[x, \mathrm{D}(y), z]_{\tau}+[x, y, D(z)]_{\tau} } & =\tau(\mathrm{D}(x))[y, z]+\tau(z)[\mathrm{D}(x), y] \\
& +\tau(y)[z, \mathrm{D}(x)]+\tau(x)[\mathrm{D}(y), z] \\
& +\tau(z)[x, \mathrm{D}(y)]+\tau(\mathrm{D}(y))[z, x] \\
& +\tau(x)[y, D(z)]+\tau(\mathrm{D}(z))[x, y] \\
& +\tau(y)[\mathrm{D}(z), x]
\end{aligned}
$$

Now, if $\mathrm{D}(\mathfrak{g}) \subset \operatorname{Ker}(\tau)$, then

$$
\begin{aligned}
& {[\mathrm{D}(x), y, z]_{\tau}+[x, \mathrm{D}(y), z]_{\tau}+[x, y, \mathrm{D}(z)]_{\tau}} \\
& =\tau(x)([\mathrm{D}(y), z]+[y, \mathrm{D}(z)])+\tau(y)([z, \mathrm{D}(x)]+[\mathrm{D}(z), x])+ \\
& \quad \tau(z)([\mathrm{D}(x), y]+[x, \mathrm{D}(y)]) \\
& =\tau(x) \mathrm{D}([y, z])+\tau(y) \mathrm{D}([z, x])+\tau(z) \mathrm{D}([x, y]) \\
& =\mathrm{D}(\tau(x)[y, z]+\tau(y)[z, x]+\tau(z)[x, y])=\mathrm{D}\left([x, y, z]_{\tau}\right) .
\end{aligned}
$$

Thus $\mathrm{D}$ is a derivation of $\left(\mathfrak{g},[\cdot, \cdot, \cdot]_{\tau}\right)$.

A more powerful criteria is given in the next theorem.

Lemma 3.8. Let $\mathrm{D}: \mathfrak{g} \rightarrow \mathfrak{g}$ be a Lie algebra derivation, then $\tau \circ \mathrm{D}$ is a trace function on $\mathfrak{g}$. 
Proof. For all $x, y \in \mathfrak{g}$, we have:

$$
\tau(D([x, y]))=\tau([D(x), y]+[x, D(y)])=\tau([D(x), y])+\tau([x, D(y)])=0 .
$$

Theorem 3.9. [1] Let $\mathrm{D}: \mathfrak{g} \rightarrow \mathfrak{g}$ be a derivation of the Lie algebra $(\mathfrak{g},[\cdot, \cdot])$, then $\mathrm{D}$ is a derivation of the induced ternary Lie algebra $\left(\mathfrak{g},[\cdot, \cdot, \cdot]_{\tau}\right)$ if and only if:

$$
[x, y, z]_{\tau \circ D}=0, \forall x, y, z \in \mathfrak{g} .
$$

Proof. Let D be a derivation of $\mathfrak{g}$ and $x, y, z \in \mathfrak{g}$ :

$$
\begin{aligned}
D\left([x, y, z]_{\tau}\right) & =\tau(x) D([y, z])+\tau(y) D([z, x])+\tau(z) D([x, y]) \\
& =\tau(x)[D(y), z]+\tau(y)[D(z), x]+\tau(z)[D(x), y] \\
& +\tau(x)[y, D(z)]+\tau(y)[z, D(x)]+\tau(z)[x, D(y)] \\
& +\tau(D(x))[y, z]+\tau(D(y))[z, x]+\tau(D(z))[x, y] \\
& -(\tau(D(x))[y, z]+\tau(D(y))[z, x]+\tau(D(z))[x, y]) \\
& =[D(x), y, z]_{\tau}+[x, D(y), z]_{\tau}+[x, y, D(z)]_{\tau}-[x, y, z]_{\tau \circ D} .
\end{aligned}
$$

Proposition 3.10. Let $\mathrm{D}$ be a derivation of $(\mathfrak{g},[\cdot, \cdot, \cdot])$. For $\mathfrak{w} \in \mathfrak{g}$, $\mathrm{D}$ is a derivation of the Lie algebra $\left(\mathfrak{g},[\cdot, \cdot]_{\mathfrak{w}}\right)$ if and only if $\mathrm{D}(\mathfrak{w}) \in \mathrm{Z}(\mathfrak{g},[\cdot, \cdot, \cdot])$.

Proof. Let $x, y \in \mathfrak{g}$,

$$
\begin{aligned}
D\left([x, y]_{w}\right) & =D([x, y, w])=[D(x), y, w]+[x, D(y), w]+[x, y, D(w)] \\
& =[D(x), y]_{w}+[x, D(y)]_{w}+[x, y, D(w)] .
\end{aligned}
$$

Hence if $\mathrm{D}(\boldsymbol{w})$ is in the center of the ternary Lie algebra $(\mathfrak{g},[\cdot, \cdot, \cdot])$, then $\mathrm{D}$ would be a derivation of the induced Lie algebra $\left(\mathfrak{g},[\cdot, \cdot]_{w}\right)$.

For any $x=\left(x_{1}, x_{2}\right) \in \mathfrak{g} \times \mathfrak{g}$, the map defined by

$$
\begin{aligned}
\operatorname{ad}_{\mathrm{x}}: \mathfrak{g} & \longrightarrow \mathfrak{g} \\
\mathfrak{u} & \mapsto\left[\mathrm{x}_{1}, \mathrm{x}_{2}, \mathfrak{u}\right]
\end{aligned}
$$

is a derivation which we call an inner derivation.

In fact, For $\mathfrak{u}, v, w \in \mathfrak{g}$,

$$
\begin{aligned}
\operatorname{ad}_{x}([u, v, w]) & =\left[x_{1}, x_{2},[u, v, w]\right] \\
& =\left[\left[x_{1}, x_{2}, u\right], v, w\right]+\left[u,\left[x_{1}, x_{2}, v\right], w\right]+\left[u, v,\left[x_{1}, x_{2}, w\right]\right] \\
& =\left[\operatorname{ad}_{x}(u), v, w\right]+\left[u, a_{x}(v), w\right]+\left[u, v, \operatorname{ad}_{x}(w)\right] .
\end{aligned}
$$




\section{Remark 3.11.}

$$
\begin{aligned}
\operatorname{ad}: \mathfrak{g} \times \mathfrak{g} & \longrightarrow \operatorname{gl}(\mathfrak{g}) \\
\left(x_{1}, \chi_{2}\right) & \mapsto \operatorname{ad}_{\left(x_{1}, x_{2}\right)}
\end{aligned}
$$

is the adjoint representation of $\mathfrak{g}$.

It turns out that all the derivations on a semisimple Lie algebra are inner derivations. This is also true for Lie triple systems (Lister 1952) and many other algebraic structures. In particular, all the derivations of the ternary Lie algebra defined in Example 2.5 are inner.

Proposition 3.12. The space Der( $\mathfrak{g})$ is an invariant of the ternary Lie algebra $\mathfrak{g}$.

Remark 3.13. Here the space of derivations is considered as ternary Lie algebra induced from the Lie algebra structure as shown in 3.4.

Proof. Let $\sigma:\left(\mathfrak{g},[\cdot, \cdot, \cdot]_{\mathfrak{g}}\right) \longrightarrow\left(\eta,[\cdot, \cdot, \cdot]_{\eta}\right)$ be a ternary Lie algebras isomorphism and let $\mathrm{D}$ be a derivation of $\mathfrak{g}$, so for any $x, y, z \in \eta$ we have:

$$
\begin{aligned}
\sigma \operatorname{D\sigma }^{-1}\left([x, y, z]_{\mathfrak{\eta}}\right) & =\sigma D\left(\left[\sigma^{-1}(x), \sigma^{-1}(y), \sigma^{-1}(z)\right]_{\mathfrak{g}}\right) \\
& =\sigma\left(\left[\operatorname{D\sigma }^{-1}(x), \sigma^{-1}(y), \sigma^{-1}(z)\right]_{\mathfrak{g}}\right)+\sigma\left(\left[\sigma^{-1}(x), \mathrm{D}^{-1}(y), \sigma^{-1}(z)\right]_{\mathfrak{g}}\right) \\
& +\sigma\left(\left[\sigma^{-1}(x), \sigma^{-1}(y), \operatorname{D\sigma }^{-1}(z)\right]_{\mathfrak{g}}\right) \\
& =\left[\sigma \operatorname{D\sigma }^{-1}(x), y, z\right]_{\eta}+\left[x, \sigma D \sigma^{-1}(y), z\right]_{\eta}+\left[x, y, \sigma D \sigma^{-1}(z)\right]_{\eta} .
\end{aligned}
$$

Thus $\sigma \mathrm{D} \sigma^{-1}$ is a derivation of $\eta$, hence the mapping

$$
\begin{aligned}
\phi: \operatorname{Der}(\mathfrak{g}) & \longrightarrow \operatorname{Der}(\eta) \\
\mathrm{D} & \longmapsto \sigma \sigma^{-1}
\end{aligned}
$$

is an isomorphism of ternary Lie algebras.

In fact, it is easy to see that $\phi$ is linear. Moreover, let $D_{1}, D_{2}, D_{3}$ be derivations of $\mathfrak{g}$ :

$$
\begin{aligned}
\phi\left(\left[\mathrm{D}_{1}, \mathrm{D}_{2}, \mathrm{D}_{3}\right]_{\mathrm{tr}}\right)= & \phi\left(\operatorname{tr}\left(\mathrm{D}_{1}\right)\left[\mathrm{D}_{2}, \mathrm{D}_{3}\right]\right)+\phi\left(\operatorname{tr}\left(\mathrm{D}_{3}\right)\left[\mathrm{D}_{1}, \mathrm{D}_{2}\right]\right)+\phi\left(\operatorname{tr}\left(\mathrm{D}_{2}\right)\left[\mathrm{D}_{3}, \mathrm{D}_{1}\right]\right) \\
= & \operatorname{tr}\left(\mathrm{D}_{1}\right) \phi\left(\left[\mathrm{D}_{2}, \mathrm{D}_{3}\right]\right)+\operatorname{tr}\left(\mathrm{D}_{3}\right) \phi\left(\left[\mathrm{D}_{1}, \mathrm{D}_{2}\right]\right)+\operatorname{tr}\left(\mathrm{D}_{2}\right) \phi\left(\left[\mathrm{D}_{3}, \mathrm{D}_{1}\right]\right) \\
= & \operatorname{tr}\left(\phi\left(\mathrm{D}_{1}\right)\right)\left[\phi\left(\mathrm{D}_{2}\right), \phi\left(\mathrm{D}_{3}\right)\right]+\operatorname{tr}\left(\phi\left(\mathrm{D}_{3}\right)\right)\left[\phi\left(\mathrm{D}_{1}\right), \phi\left(\mathrm{D}_{2}\right)\right] \\
& +\operatorname{tr}\left(\phi\left(\mathrm{D}_{2}\right)\right)\left[\phi\left(\mathrm{D}_{3}\right), \phi\left(\mathrm{D}_{1}\right)\right]
\end{aligned}
$$

since $\phi$ is a morphism of the Lie algebras $\operatorname{Der}(\mathfrak{g})$ and $\operatorname{Der}(\eta)$, and $\operatorname{tr}(\mathrm{D})=$ $\operatorname{tr}\left(\sigma D \sigma^{-1}\right)$. Then $\phi\left(\left[D_{1}, D_{2}, D_{3}\right]_{t r}\right)=\left[\phi\left(D_{1}\right), \phi\left(D_{2}\right), \phi\left(D_{3}\right)\right]_{t r}$. 
Definition 3.14. The centroid of a ternary Lie algebra $\mathfrak{g}$ is the set of all linear maps D that satisfies:

$$
\mathrm{D}([x, y, z])=[\mathrm{D}(\mathrm{x}), \mathrm{y}, z]=[x, \mathrm{D}(\mathrm{y}), z]=[x, y, D(z)],
$$

for all $x, y, z \in \mathfrak{g}$. We denote by $\mathrm{C}(\mathfrak{g})$ the centroid of $\mathfrak{g}$.

Remark 3.15. We can define the centroid only by the equality $\mathrm{D}([\mathrm{x}, \mathrm{y}, z])=$ $[\mathrm{D}(\mathrm{x}), \mathrm{y}, z]$, and the two other follows by the skew symmetry of the bracket.

Proposition 3.16. The centroid of a ternary Lie algebra $\mathfrak{g}$ is a ternary Lie subalgebra of $\left(\operatorname{Der}(\mathfrak{g}),[\cdot, \cdot, \cdot]_{\mathrm{tr}}\right)$.

Proof. Let $\mathrm{D}_{1}, \mathrm{D}_{2}, \mathrm{D}_{3} \in \mathrm{C}(\mathfrak{g})$ and $\psi=\left[\mathrm{D}_{1}, \mathrm{D}_{2}, \mathrm{D}_{3}\right]_{\mathrm{tr}}$. For simplicity we let $\lambda_{i}=\operatorname{tr}\left(D_{i}\right)$ for $i=1,2,3$. So $\psi=\lambda_{1}\left(D_{2} D_{3}-D_{3} D_{2}\right)+\lambda_{3}\left(D_{1} D_{2}-D_{2} D_{1}\right)+$ $\lambda_{2}\left(D_{3} D_{1}-D_{1} D_{3}\right)$. Then for $x, y, z \in \mathfrak{g}$, we have

$$
\begin{aligned}
{[\psi(x), y, z] } & =\left[\lambda_{1}\left(D_{2} D_{3}-D_{3} D_{2}\right)(x)+\lambda_{3}\left(D_{1} D_{2}-D_{2} D_{1}\right)(x)\right. \\
& \left.+\lambda_{2}\left(D_{3} D_{1}-D_{1} D_{3}\right)(x), y, z\right] \\
& =\lambda_{1}\left(\left[D_{2} D_{3}(x), y, z\right]-\left[D_{3} D_{2}(x), y, z\right]\right)+\lambda_{3}\left(\left[D_{1} D_{2}(x), y, z\right]\right. \\
& \left.-\left[D_{2} D_{1}(x), y, z\right]\right)+\lambda_{2}\left(\left[D_{3} D_{1}(x), y, z\right]-\left[D_{1} D_{3}(x), y, z\right]\right) \\
& =\lambda_{1}\left(D_{2}\left(\left[D_{3}(x), y, z\right]\right)-D_{3}\left(\left[D_{2}(x), y, z\right]\right)\right)+\lambda_{3}\left(D_{1}\left(\left[D_{2}(x), y, z\right]\right)\right. \\
& \left.-D_{2}\left(\left[D_{1}(x), y, z\right]\right)\right)+\lambda_{2}\left(D_{3}\left(\left[D_{1}(x), y, z\right]\right)-D_{1}\left(\left[D_{3}(x), y, z\right]\right)\right) \\
& =\lambda_{1}\left(D_{2} D_{3}-D_{3} D_{2}\right)([x, y, z])+\lambda_{3}\left(D_{1} D_{2}-D_{2} D_{1}\right)([x, y, z])+ \\
& \lambda_{2}\left(D_{3} D_{1}-D_{1} D_{3}\right)([x, y, z]) . \\
& =\psi([x, y, z]) .
\end{aligned}
$$

Proposition 3.17. Let $\mathrm{D} \in \mathrm{C}(\mathfrak{g},[\cdot, \cdot])$. If for every $\mathfrak{u}, \boldsymbol{v} \in \mathfrak{g}$ we have

$$
\tau(u) D(v)=\tau(D(u)) v
$$

then $\mathrm{D} \in \mathrm{C}\left(\mathfrak{g},[\cdot, \cdot, \cdot]_{\tau}\right)$.

Proof.

$$
\begin{aligned}
\mathrm{D}\left([x, y, z]_{\tau}\right) & =\tau(x) \mathrm{D}([y, z])+\tau(z) \mathrm{D}([x, y])+\tau(y) \mathrm{D}([z, x]) \\
& =\tau(\mathrm{D}(x))[y, z]+\tau(z)[\mathrm{D}(x), y]+\tau(y)[\mathrm{D}(z), x] \\
& =\tau(\mathrm{D}(x))[y, z]+\tau(z)[\mathrm{D}(x), y]+\tau(y)[z, D(x)] \\
& =[\mathrm{D}(x), y, z]_{\tau} .
\end{aligned}
$$


On the other hand, any centroid element of a ternary Lie algebra $(\mathfrak{g},[\cdot, \cdot, \cdot])$ is a centroid element of an induced Lie algebra $\left(\mathfrak{g},[\cdot, \cdot]_{w}\right)$.

The following proposition reduces the centroid of any simple ternary Lie algebra to the space of its homothety.

Proposition 3.18. Let $(\mathfrak{g},[\cdot, \cdot, \cdot])$ be a simple ternary Lie algebra over an algebraically closed field $\mathbb{K}$. We have

$$
\mathrm{C}(\mathfrak{g})=\mathbb{K} \mathrm{Id},
$$

where Id is the identity map on $\mathfrak{g}$.

Proof. First, one can see that the adjoint representation of $\mathfrak{g}$ is simple because otherwise, if a subset $\mathcal{A}$ of $\mathfrak{g}$ is stable under the action of $\operatorname{ad}_{\left(\chi_{1}, \chi_{2}\right)}$ for any $\chi_{1}, \chi_{2} \in \mathfrak{g}$, then $\mathcal{A}$ is an ideal.

In addition, for any centroid element $\mathrm{D}$ we have

$$
\mathrm{D}([x, y, z])=[x, y, D(z)] .
$$

Therefore

$$
\mathrm{D} \circ \operatorname{ad}_{(x, y)}=\operatorname{ad}_{(x, y)} \circ \mathrm{D} .
$$

Thus using the Schur Lemma, we conclude that $\mathrm{D}=\lambda \mathrm{Id}$ for some scalar $\lambda$.

Definition 3.19. A linear map $\mathrm{D}$ is a central derivation of $(\mathfrak{g},[\cdot, \cdot, \cdot])$ if $\mathrm{D}(\mathfrak{g}) \subset \mathrm{Z}(\mathfrak{g})$ and $\mathrm{D}\left(\mathfrak{g}^{1}\right)=\{0\}$.

We denote by $Z \operatorname{Der}(\mathfrak{g})$ the set of all central derivations of $(\mathfrak{g},[\cdot, \cdot, \cdot])$.

Example 3.20. A simple ternary Lie algebra does not have a non zero central derivation since $\mathfrak{g}^{1}=\mathfrak{g}$.

Proposition 3.21. For a ternary Lie algebra $(\mathfrak{g},[\cdot, \cdot, \cdot])$, we have

$$
\operatorname{ZDer}(\mathfrak{g})=\operatorname{Der}(\mathfrak{g}) \cap \mathrm{C}(\mathfrak{g}) .
$$

Proof. A central derivation is obviously a derivation of the ternary Lie algebra. Moreover, it is a centroid element since

$$
\mathrm{D}([\mathrm{x}, \mathrm{y}, z])=[\mathrm{D}(\mathrm{x}), \mathrm{y}, z]=[\mathrm{x}, \mathrm{D}(\mathrm{y}), z]=[\mathrm{x}, \mathrm{y}, \mathrm{D}(z)]=0
$$

for all $x, y, z \in \mathfrak{g}$. Conversely, if $\mathrm{D} \in \operatorname{Der}(\mathfrak{g}) \cap \mathrm{C}(\mathfrak{g})$, then

$$
\mathrm{D}([x, y, z])=3 \mathrm{D}([x, y, z]) .
$$

Therefore $D([x, y, z])=0$. Also $[D(x), y, z]=3[D(x), y, z]$, thus $[D(x), y, z]=0$ and $\mathrm{D}(\mathrm{x}) \in \mathrm{Z}(\mathfrak{g})$. 
Here again we study central derivations of a ternary Lie algebra induced by a Lie algebra and vice versa.

Proposition 3.22. Let $(\mathfrak{g},[\cdot, \cdot, \cdot])$ be a ternary Lie algebra. For any $\mathfrak{w} \in \mathfrak{g}$, let $\left(\mathfrak{g},[\cdot, \cdot]_{w}\right)$ be the induced Lie algebra. Every Central derivation of $(\mathfrak{g},[\cdot, \cdot, \cdot])$ is also a central derivation of the induced $\left(\mathfrak{g},[\cdot, \cdot]_{w}\right)$.

Proof. The proof of is straightforward under the remark that $\mathbf{Z}(\mathfrak{g},[\cdot, \cdot, \cdot]) \subset \mathbf{Z}\left(\mathfrak{g},[\cdot, \cdot]_{w}\right)$, and $[\mathfrak{g}, \mathfrak{g}]_{w} \subset[\mathfrak{g}, \mathfrak{g}, \mathfrak{g}]$.

Proposition 3.23. Let $\left(\mathfrak{g},[\cdot, \cdot, \cdot]_{\tau}\right)$ be a ternary Lie algebra induced by a Lie algebra $(\mathfrak{g},[\cdot, \cdot])$ and the trace map $\tau$. Let $\mathrm{D} \in \operatorname{ZDer}(\mathfrak{g},[\cdot, \cdot]), \mathrm{D}$ is a central derivation of $\left(\mathfrak{g},[\cdot, \cdot, \cdot]_{\tau}\right)$ if and only if $\mathrm{D}(\mathfrak{g}) \subset \operatorname{Ker}(\tau)$.

Proof. Let $\mathrm{D} \in \operatorname{ZDer}(\mathfrak{g},[\cdot, \cdot])$ and $x, y, z \in \mathfrak{g}$,

$$
\mathrm{D}\left([x, y, z]_{\tau}\right)=\tau(x) \mathrm{D}([y, z])+\tau(z) \mathrm{D}([x, y])+\tau(y) \mathrm{D}([z, x])=0 .
$$

In addition,

$$
[D(x), y, z]_{\tau}=\tau(D(x))[y, z]+\tau(z)[D(x), y]+\tau(y)[z, D(x)]=\tau(D(x))[y, z] .
$$

\subsection{Quasi-derivations and quasi-centroids.}

Definition 3.24. A linear map D on $\mathfrak{g}$ is a quasi-derivation if there exists $D^{\prime}$ such that

$$
[D(x), y, z]+[x, D(y), z]+[x, y, D(z)]=D^{\prime}([x, y, z]),
$$

for all $x, y, z \in \mathfrak{g}$. We denote by $\operatorname{QDer}(\mathfrak{g})$ the set of all quasi-derivations of $\mathfrak{g}$.

Example 3.25. Let $(\mathrm{V},[\cdot, \cdot, \cdot])$ be the ternary Lie algebra defined in Example 2.5. For any linear map $\mathrm{D}$ of $\mathrm{V}$ with $\mathrm{M}=\left(\mathrm{a}_{\mathrm{ij}}\right)$ its matrix in the base $\left(\mathrm{e}_{1}, \mathrm{e}_{2}, \mathrm{e}_{3}, \mathrm{e}_{4}\right)$, there exists $\mathrm{D}^{\prime}$ such that $\mathrm{D} \in \mathrm{QDer}(\mathrm{V})$ and the matrix $\mathrm{M}^{\prime}=\left(\mathrm{b}_{i j}\right)$ of $\mathrm{D}^{\prime}$ is given by:

$$
b_{i j}=-a_{j i} \quad \text { for } 1 \leq i \neq j \leq 4
$$

and

$$
b_{i i}=\sum_{\substack{j=1 \\ j \neq i}}^{4} a_{j j} .
$$

As in Theorem 3.9, the next proposition establish the link between the quasiderivations of a Lie algebra and the induced ternary Lie algebra. 
Proposition 3.26. Let $\mathrm{D}$ be a quasi-derivation of a Lie algebra $(\mathfrak{g},[\cdot, \cdot])$ and let $\tau$ be a trace function on $\mathfrak{g} . \mathrm{D}$ is a quasi-derivation of $\left(\mathfrak{g},[\cdot, \cdot, \cdot]_{\tau}\right)$ if and only if $[x, y, z]_{\tau \circ D}=0$ for any $x, y, z$.

Remark 3.27. Unlike Lemma 3.8, in this case the map $\tau \circ \mathrm{D}$ is not necessarily a trace on $\mathfrak{g}$.

Every derivation is obviously a quasi-derivation, so we have $\operatorname{Der}(\mathfrak{g}) \subset \operatorname{QDer}(\mathfrak{g})$. We will see now that a sum of any derivation and a centroid element is also a quasi-derivation:

Proposition 3.28. If $(\mathfrak{g},[\cdot, \cdot, \cdot])$ is a ternary Lie algebra with trivial center, then we have

$$
\operatorname{Der}(\mathfrak{g}) \oplus \mathrm{C}(\mathfrak{g}) \subset \mathrm{QDer}(\mathfrak{g})
$$

Proof. Let $\mathrm{D} \in \mathrm{C}(\mathfrak{g})$, for any $x, y, z \in \mathfrak{g}$, we have

$$
[\mathrm{D}(\mathrm{x}), \mathrm{y}, z]+[x, \mathrm{D}(\mathrm{y}), z]+[x, y, D(z)]=3 \mathrm{D}([x, y, z]) .
$$

So D is a quasi-derivation, thus $\operatorname{Der}(\mathfrak{g})+\mathrm{C}(\mathfrak{g}) \subset \operatorname{QDer}(\mathfrak{g})$. Now if $\mathrm{D} \in \operatorname{Der}(\mathfrak{g}) \cap$ $\mathrm{C}(\mathfrak{g})$, then

$$
[D(x), y, z]+[D(x), y, z]+[D(x), y, z]=[D(x), y, z] .
$$

Thus $[D(x), y, z]=0$, therefore $D(x) \in Z(\mathfrak{g})$, for every $x$, which means that $D=0$. Hence $\operatorname{Der}(\mathfrak{g}) \cap \mathrm{C}(\mathfrak{g})=\{0\}$.

Proposition 3.29. Let $(\mathfrak{g},[\cdot, \cdot, \cdot])$ be a ternary Lie algebra with trivial center and suppose that $\mathfrak{g}=\mathcal{I} \oplus \mathcal{J}$, then

(1) $\operatorname{QDer}(\mathfrak{g})=\mathrm{QDer}(\mathcal{I}) \oplus \operatorname{QDer}(\mathcal{J})$

(2) $\mathrm{C}(\mathfrak{g})=\mathrm{C}(\mathcal{I}) \oplus \mathrm{C}(\mathcal{J})$.

Proof. Since Lemma 3.6 can be applied to any quasi-derivation and any centroid element, so the decomposition in Proposition 3.5 holds naturally.

Definition 3.30. The quasi-centroid of $\mathfrak{g}$ is the set of all linear maps D such that:

$$
[\mathrm{D}(\mathrm{x}), \mathrm{y}, z]=[x, \mathrm{D}(\mathrm{y}), z]=[x, y, D(z)],
$$

for all $x, y, z \in \mathfrak{g}$. We denote by $\mathrm{QC}(\mathfrak{g})$ the centroid of $\mathfrak{g}$.

Proposition 3.31. Let $\mathrm{D} \in \mathrm{QC}(\mathfrak{g},[\cdot, \cdot])$. Suppose that $\mathrm{D}(\mathfrak{g}) \subset \operatorname{Ker}(\tau)$ and for every $\mathfrak{u}, v \in \mathfrak{g}$ we have $\tau(\mathfrak{u}) . v=\tau(v) . u$, then $\mathrm{D} \in \mathrm{QC}\left(\mathfrak{g},[\cdot, \cdot, \cdot]_{\tau}\right)$.

The proof is quite similar to the proof of Proposition 3.17. 
Lemma 3.32. The derived algebra $\mathfrak{g}^{1}$ is preserved under $\operatorname{Der}(\mathfrak{g})$ and $\mathrm{C}(\mathfrak{g})$ but not $\mathrm{QC}(\mathfrak{g})$.

Proof. If $\mathrm{D}$ is a derivation of $\mathfrak{g}$, then by definition, for any $x, y, z \in \mathfrak{g}$ we have

$$
\mathrm{D}([x, y, z])=[\mathrm{D}(\mathrm{x}), \mathrm{y}, z]+[x, \mathrm{D}(\mathrm{y}), z]+[x, y, D(z)] \in \mathfrak{g}^{1} .
$$

Similarly, if $D \in C(\mathfrak{g})$, so $D([x, y, z])=[D(x), y, z] \in \mathfrak{g}^{1}$.

We show that the derived algebra is not generally preserved by the quasi-centroid, by the following counter example. Let $(\mathrm{L},[\cdot, \cdot, \cdot])$ be the ternary Lie algebra with the basis $\left\{e_{1}, e_{2}, e_{3}\right\}$ such that $\left[e_{1}, e_{2}, e_{3}\right]=e_{1}$. Let $\varphi$ be a linear map defined by $\varphi\left(e_{1}\right)=e_{2}$ and $\varphi\left(e_{e}\right)=\varphi\left(e_{3}\right)=0$. Then $\varphi \in \mathrm{QC}(\mathrm{L})$ since $\left[\varphi\left(e_{1}\right), e_{2}, e_{3}\right]+$ $\left[e_{1}, \varphi\left(e_{2}\right), e_{3}\right]+\left[e_{1}, e_{2}, \varphi\left(e_{3}\right)\right]=0$. However $\varphi$ does not preserve $L^{1}=<e_{1}>$.

3.3. $(\alpha, \beta, \gamma, \theta)$-Derivations and $(\alpha, \beta, \gamma, \theta)$-quasiderivations. We will now define another generalization for a derivation of $(\mathfrak{g},[\cdot, \cdot, \cdot])$.

Definition 3.33. Let $\mathfrak{g}$ be a ternary Lie algebra, $\mathrm{D} \in \operatorname{End}(\mathfrak{g})$ and let $\alpha, \beta, \gamma, \theta \in \mathbb{K}$. We say that $\mathrm{D}$ is an $(\alpha, \beta, \gamma, \theta)$-derivation of $\mathfrak{g}$ if:

$$
\alpha \mathrm{D}\left(\left[\mathrm{x}_{1}, x_{2}, x_{3}\right]\right)=\beta\left[\mathrm{D}\left(\mathrm{x}_{1}\right), \mathrm{x}_{2}, \mathrm{x}_{3}\right]+\gamma\left[\mathrm{x}_{1}, \mathrm{D}\left(\mathrm{x}_{2}\right), \mathrm{x}_{3}\right]+\theta\left[\mathrm{x}_{1}, \mathrm{x}_{2}, \mathrm{D}\left(\mathrm{x}_{3}\right)\right],
$$

for any $x_{1}, x_{2}, x_{3} \in \mathfrak{g}$.

We denote by $\mathrm{D}(\alpha, \beta, \gamma, \theta)$ the set of $(\alpha, \beta, \gamma, \theta)$-derivations.

Remark 3.34. It is clear that $\mathrm{D}(0,0,0,0)=\operatorname{End}(\mathfrak{g})$, therefore we can assume that the parameters $\alpha, \beta, \gamma, \theta$ are not all zeros. One can also see that $\mathrm{D}(1,1,1,1)=$ $\operatorname{Der}(\mathfrak{g})$.

Theorem 3.35. Suppose that $\alpha \neq 0$. The space $\mathrm{D}(\alpha, \beta, \gamma, \theta)$ is one of the following spaces:

- $\mathrm{D}(1, \lambda, 0,0)$

- $\mathrm{D}(1, \lambda, \delta, \delta)$

- $\mathrm{D}(1, \lambda, 0,0) \cap \mathrm{QC}(\mathfrak{g})$

for some $\lambda, \delta \in \mathbb{K}$.

Proof. Let $\mathrm{D} \in \mathrm{D}(\alpha, \beta, \gamma, \theta)$. Using the skew-symmetry of the bracket we have the following equalities, for any $x, y, z \in \mathfrak{g}$

$$
\begin{array}{r}
\alpha \mathrm{D}([x, y, z])=\beta[D(x), y, z]+\gamma[x, D(y), z]+\theta[x, y, D(z)] \\
\alpha \mathrm{D}([x, y, z])=\beta[D(z), x, y]+\gamma[z, D(x), y]+\theta[z, x, D(y)] \\
-\alpha D([x, y, z])=\beta[D(x), z, y]+\gamma[x, D(z), y]+\theta[x, z, D(y)]
\end{array}
$$




$$
-\alpha D([x, y, z])=\beta[D(y), x, z]+\gamma[y, D(x), z]+\theta[y, x, D(z)] .
$$

Now by adding eq (3) to each equation, we get

$$
\begin{aligned}
& 0=(\gamma-\theta)[x, D(y), z]+(\theta-\gamma)[x, y, D(z)] \\
& 0=(\beta-\gamma)[D(x), y, z]+(\gamma-\beta)[x, D(y), z]
\end{aligned}
$$

If $\gamma=\theta=0$, then $D \in D\left(1, \frac{\beta}{\alpha}, 0,0\right)$. If $\gamma=\theta \neq 0, D \in D\left(1, \frac{\beta}{\alpha}, \frac{\gamma}{\alpha}, \frac{\gamma}{\alpha}\right)$. Similarly, if $\beta=\gamma$, then $\mathrm{D}(\alpha, \beta, \gamma, \theta)=\mathrm{D}\left(1, \frac{\theta}{\alpha}, 0,0\right)$ or $\mathrm{D}(\alpha, \beta, \gamma, \theta)=\mathrm{D}\left(1, \frac{\gamma}{\alpha}, \frac{\gamma}{\alpha}, \frac{\theta}{\alpha}\right)$. If $\theta \neq \gamma$ and $\beta \neq \gamma$, it follows from the equations above that $\mathrm{D} \in \mathrm{QC}(\mathfrak{g})$ hence $\mathrm{D}$ satisfies $\mathrm{D}([x, y, z])=\frac{\beta+\gamma+\theta}{\alpha}[\mathrm{D}(x), y, z]$.

Let us now discuss the case of a ternary Lie algebra induced by a Lie algebra. Here we denote the space of the $(\alpha, \beta, \gamma, \theta)$-derivations of the induced ternary Lie algebra $\left(\mathfrak{g},[\cdot, \cdot, \cdot]_{\tau}\right)$ by $\mathrm{D}_{\tau}(\alpha, \beta, \gamma, \theta)$.

Proposition 3.36. Suppose that for every $\mathfrak{u}, v \in \mathfrak{g}$ we have $\tau(\mathfrak{u}) . v=\tau(v) . u$, then any $(\alpha, \beta, \gamma)$-derivation $\mathrm{D}$ of $(\mathfrak{g},[\cdot, \cdot])$ that satisfies $\mathrm{D}(\mathfrak{g}) \subset \operatorname{Ker}(\tau)$ is an $\left(\alpha^{\prime}, \beta^{\prime}, \gamma^{\prime}, \theta^{\prime}\right)$-derivation of $\left(\mathfrak{g},[\cdot, \cdot, \cdot]_{\tau}\right)$ for some $\alpha^{\prime}, \beta^{\prime}, \gamma^{\prime}, \theta^{\prime}$. Precisely, D is in one of the following spaces:

(i) $\operatorname{End}(\mathfrak{g})$,

(ii) $\left\{\mathrm{f} \in \operatorname{End}(\mathfrak{g}) ; \mathfrak{f}\left(\mathfrak{g}^{1}\right)=\{0\}\right\}$,

(iii) $\mathrm{QC}\left(\mathfrak{g},[\cdot, \cdot, \cdot]_{\tau}\right)$,

(iv) $\mathrm{QC}\left(\mathfrak{g},[\cdot, \cdot, \cdot]_{\mathcal{\tau}}\right) \cap\left\{\mathrm{f} \in \operatorname{End}(\mathfrak{g}) ; \mathfrak{f}\left(\mathfrak{g}^{1}\right)=\{0\}\right\}$,

(v) $\mathrm{D}_{\tau}(\delta, 1,1,1)$, for some $\delta \in \mathrm{K}$,

(vi) $\mathrm{QC}\left(\mathfrak{g},[\cdot, \cdot, \cdot]_{\tau}\right) \cap \mathrm{D}_{\tau}(\delta, 1,1,1)$, for some $\delta \in \mathrm{K}$.

To prove this, we recall the following proposition stated in [13].

Proposition 3.37. Let $\mathfrak{g}$ be a Lie algebra and $\alpha, \beta, \gamma \in \mathbb{C} . \mathrm{D}(\alpha, \beta, \gamma)$ is one of the following spaces:

(i) $\mathrm{D}(0,0,0)=\operatorname{End}(\mathfrak{g})$,

(ii) $\mathrm{D}(1,0,0)=\left\{\mathrm{f} \in \operatorname{End}(\mathfrak{g}) ; f\left(\mathfrak{g}^{1}\right)=\{0\}\right\}$,

(iii) $\mathrm{D}(0,1,-1)=\mathrm{QC}\left(\mathfrak{g},[\cdot, \cdot, \cdot]_{\tau}\right)$,

(iv) $\mathrm{D}(1,1,-1)=\mathrm{QC}(\mathfrak{g},[\cdot, \cdot]) \cap\left\{\mathfrak{f} \in \operatorname{End}(\mathfrak{g}) ; \mathfrak{f}\left(\mathfrak{g}^{1}\right)=\{0\}\right\}$,

(v) $\mathrm{D}(\delta, 1,1)$

(vi) $\mathrm{D}(\delta, 1,0)=\mathrm{QC}(\mathfrak{g},[\cdot, \cdot]) \cap \mathrm{D}(2 \delta, 1,1)$.

Proof of Proposition 3.36. (i), (ii) are obvious.

(iii), (iv) by Proposition 3.31. 
(v) Let $\mathrm{D} \in \mathrm{D}(\delta, 1,1)$, then

$$
\begin{aligned}
\delta \mathrm{D}\left([x, y, z]_{\tau}\right) & =\tau(x) \delta \mathrm{D}([y, z])+\tau(z) \delta \mathrm{D}([x, y])+\tau(y) \delta \mathrm{D}([z, x]) \\
& =\tau(x)([\mathrm{D}(y), z]+[y, D(z)])+\tau(z)([\mathrm{D}(x), y]+[x, D(y)]) \\
& +\tau(y)([D(z), x]+[z, D(x)]) \\
& =\tau(z)[D(x), y]+\tau(y)[z, D(x)]+\tau(x)[D(y), z]+\tau(z)[x, D(y)] \\
& +\tau(y)[D(z), x]+\tau(x)[y, D(z)] \\
& =[D(x), y, z]_{\tau}+[x, D(y), z]_{\tau}+[x, y, D(z)]_{\tau} .
\end{aligned}
$$

(vi) We apply $(v)$ to the space $\mathrm{D}(2 \delta, 1,1)$.

\section{Derivations and central derivations of ternary Lie algebras of dimension less or equal than 4}

In this section, we will use classification theorem of ternary Lie algebras of dimension less or equal 4 given in [5] to determine the spaces $\operatorname{Der}(\mathfrak{g})$ and $\mathrm{C}(\mathfrak{g})$. Then we conclude with the space of central derivations $Z \operatorname{Der}(\mathfrak{g})$ using Proposition 3.21.

Theorem 4.1. [5] Let $\mathfrak{g}$ be a ternary Lie algebra of dimension less or equal than 4 and let $\left(e_{\mathfrak{i}}\right)_{1 \leq \mathfrak{i} \leq \operatorname{dim}(\mathfrak{g})}$ be a basis of $\mathfrak{g}$. Then $\mathfrak{g}$ is isomorphic to one of the following:

(1) If $\operatorname{dim} \mathfrak{g}<3$, then $\mathfrak{g}$ is abelian.

(2) If $\operatorname{dim} \mathfrak{g}=3$, then

a. $\mathfrak{g}$ is abelian.

b. $\left[e_{1}, e_{2}, e_{3}\right]=e_{1}$.

(3) If $\operatorname{dim} \mathfrak{g}=4$, then

a. $\mathfrak{g}$ is abelian.

b. $\left[e_{2}, e_{3}, e_{4}\right]=e_{1}$.

c. $\left[e_{1}, e_{2}, e_{3}\right]=e_{1}$.

d. $\left[e_{1}, e_{2}, e_{4}\right]=\alpha e_{3}+\beta e_{4} ;\left[e_{1}, e_{2}, e_{3}\right]=\gamma e_{3}+\delta e_{4}$, where $\left(\begin{array}{cc}\alpha & \beta \\ \gamma & \delta\end{array}\right)$ is an invertible matrix.

e. $\left[e_{2}, e_{3}, e_{4}\right]=e_{1} ;\left[e_{1}, e_{3}, e_{4}\right]=\alpha e_{2} ;\left[e_{1}, e_{2}, e_{4}\right]=\beta e_{3}$ with $\alpha, \beta \neq 0$.

f. $\left[e_{2}, e_{3}, e_{4}\right]=e_{1} ;\left[e_{1}, e_{3}, e_{4}\right]=\alpha e_{2} ;\left[e_{1}, e_{2}, e_{4}\right]=\beta e_{3} ;\left[e_{1}, e_{2}, e_{3}\right]=\gamma e_{4}$ with $\alpha, \beta, \gamma \neq 0$.

The omitted brackets are either zeros or can be obtained by skew-symmetry.

Let $D$ be a linear map on $\mathfrak{g}$ and let $M=\left(a_{i, j}\right)$ its matrix in the basis $\left(e_{i}\right)_{1 \leq i \leq \operatorname{dim}(\mathfrak{g})}$. We will compute the spaces of derivations. Each of the following items corresponds to its respective case in the previous theorem. 
(1) If $\operatorname{dim}(\mathfrak{g})<3$, then we have

$$
\operatorname{Der}(\mathfrak{g})=\mathrm{C}(\mathfrak{g})=\operatorname{ZDer}(\mathfrak{g})=\operatorname{End}(\mathfrak{g})
$$

(2) $\operatorname{dim}(\mathfrak{g})=3,\left\{e_{1}, e_{2}, e_{3}\right\}$ a basis of $\mathfrak{g}$

a. $\mathfrak{g}$ is abelian: same as the case (1).

b. $\left[e_{1}, e_{2}, e_{3}\right]=e_{1}$. So

$* \operatorname{Der}(\mathfrak{g})=\{\mathrm{D} \in \operatorname{End}(\mathfrak{g})$ such that,

$$
\left.M=\left(\begin{array}{ccc}
a_{11} & a_{12} & a_{13} \\
0 & a_{22} & a_{23} \\
0 & a_{32} & -a_{22}
\end{array}\right)\right\}
$$

* $\mathrm{C}(\mathfrak{g})=\{\mathrm{D} \in \operatorname{End}(\mathfrak{g})$ such that,

$$
\left.M=\left(\begin{array}{ccc}
a_{11} & a_{12} & a_{13} \\
0 & a_{11} & a_{23} \\
0 & a_{32} & a_{11}
\end{array}\right)\right\}
$$

* Thus, $\operatorname{ZDer}(\mathfrak{g})=\{\mathrm{D} \in \operatorname{End}(\mathfrak{g})$ such that

$$
\left.M=\left(\begin{array}{ccc}
0 & a_{12} & a_{13} \\
0 & 0 & a_{23} \\
0 & a_{32} & 0
\end{array}\right)\right\}
$$

(3) $\operatorname{dim}(\mathfrak{g})=4,\left\{\boldsymbol{e}_{1}, e_{2}, e_{3}, e_{4}\right\}$ a basis of $\mathfrak{g}$

a. $\mathfrak{g}$ is abelian: same as the case (1).

b. $\left[e_{2}, e_{3}, e_{4}\right]=e_{1}$

$* \operatorname{Der}(\mathfrak{g})=\{\mathrm{D} \in \operatorname{End}(\mathfrak{g})$ such that,

$$
\left.M=\left(\begin{array}{cccc}
a_{22}+a_{33}+a_{44} & a_{12} & a_{13} & a_{14} \\
0 & a_{22} & a_{23} & a_{24} \\
0 & a_{32} & a_{33} & a_{34} \\
0 & a_{42} & a_{43} & a_{44}
\end{array}\right)\right\}
$$

* $C(\mathfrak{g})=\{\mathrm{D} \in \operatorname{End}(\mathfrak{g})$ such that,

$$
\left.M=\left(\begin{array}{cccc}
a_{11} & a_{12} & a_{13} & a_{14} \\
0 & a_{11} & a_{23} & a_{24} \\
0 & a_{32} & a_{11} & a_{34} \\
0 & a_{42} & a_{43} & a_{11}
\end{array}\right)\right\}
$$


* Then, $\operatorname{ZDer}(\mathfrak{g})=\{\mathrm{D} \in \operatorname{End}(\mathfrak{g})$ such that

$$
\left.M=\left(\begin{array}{cccc}
0 & a_{12} & a_{13} & a_{14} \\
0 & 0 & a_{23} & a_{24} \\
0 & a_{32} & 0 & a_{34} \\
0 & a_{42} & a_{43} & 0
\end{array}\right)\right\}
$$

c. $\left[e_{1}, e_{2}, e_{3},\right]=e_{1}$

$* \operatorname{Der}(\mathfrak{g})=\{\mathrm{D} \in \operatorname{End}(\mathfrak{g})$ such that,

$$
\left.M=\left(\begin{array}{cccc}
a_{11} & a_{12} & a_{13} & 0 \\
0 & a_{22} & a_{23} & 0 \\
0 & a_{32} & -a_{22} & 0 \\
0 & a_{42} & a_{43} & a_{44}
\end{array}\right)\right\}
$$

$* C(\mathfrak{g})=\{\mathrm{D} \in \operatorname{End}(\mathfrak{g})$ such that,

$$
\left.M=\left(\begin{array}{cccc}
a_{11} & a_{12} & a_{13} & a_{14} \\
0 & a_{11} & a_{23} & a_{24} \\
0 & a_{32} & a_{11} & a_{34} \\
0 & a_{42} & a_{43} & a_{44}
\end{array}\right)\right\}
$$

* Then, $\operatorname{ZDer}(\mathfrak{g})=\{\mathrm{D} \in \operatorname{End}(\mathfrak{g})$ such that

$$
\left.M=\left(\begin{array}{cccc}
0 & a_{12} & a_{13} & 0 \\
0 & 0 & a_{23} & 0 \\
0 & a_{32} & 0 & 0 \\
0 & a_{42} & a_{43} & a_{44}
\end{array}\right)\right\} .
$$

d. $\left[e_{1}, e_{2}, e_{4}\right]=\alpha e_{3}+\beta e_{4} ;\left[e_{1}, e_{2}, e_{3}\right]=\gamma e_{3}+\delta e_{4}$, with $\left(\begin{array}{cc}\alpha & \beta \\ \gamma & \delta\end{array}\right)$ is an invertible matrix.

The matrix of a derivation $\mathrm{D}$ is of the form

$$
M=\left(\begin{array}{ll}
A & 0 \\
B & C
\end{array}\right)
$$

where $A, B, C$ are $2 \times 2$ matrices such that $\operatorname{Tr}(A)=0$ except if $\beta \neq \delta$.

$* \operatorname{ZDer}(\mathfrak{g})=\{\mathrm{D} \in \operatorname{End}(\mathfrak{g})$ such that

$$
\left.M=\left(\begin{array}{cccc}
0 & a_{12} & 0 & 0 \\
a_{21} & 0 & 0 & 0 \\
a_{31} & a_{32} & 0 & 0 \\
a_{41} & a_{42} & 0 & 0
\end{array}\right)\right\}
$$


e. $\left[e_{2}, e_{3}, e_{4}\right]=e_{1} ;\left[e_{1}, e_{3}, e_{4}\right]=\alpha e_{2} ;\left[e_{1}, e_{2}, e_{4}\right]=\beta e_{3}$

$* \operatorname{Der}(\mathfrak{g})=\{\mathrm{D} \in \operatorname{End}(\mathfrak{g})$ such that,

$$
\left.M=\left(\begin{array}{cccc}
a_{11} & a_{12} & a_{13} & a_{14} \\
\alpha a_{12} & a_{11} & a_{23} & a_{24} \\
-\beta a_{13} & \frac{\beta}{\alpha} a_{23} & a_{11} & a_{34} \\
0 & 0 & 0 & -a_{11}
\end{array}\right)\right\}
$$

$* C(\mathfrak{g})=\{\mathrm{D} \in \operatorname{End}(\mathfrak{g})$ such that,

$$
\left.M=\left(\begin{array}{cccc}
a_{11} & 0 & 0 & a_{14} \\
0 & a_{11} & 0 & a_{24} \\
0 & 0 & a_{11} & a_{34} \\
0 & 0 & 0 & a_{11}
\end{array}\right)\right\}
$$

* Then, $\operatorname{ZDer}(\mathfrak{g})=\{\mathrm{D} \in \operatorname{End}(\mathfrak{g})$ such that

$$
\left.M=\left(\begin{array}{cccc}
0 & 0 & 0 & a_{14} \\
0 & 0 & 0 & a_{24} \\
0 & 0 & 0 & a_{34} \\
0 & 0 & 0 & 0
\end{array}\right)\right\}
$$

f. $\left[e_{2}, e_{3}, e_{4}\right]=e_{1} ;\left[e_{1}, e_{3}, e_{4}\right]=\alpha e_{2} ;\left[e_{1}, e_{2}, e_{4}\right]=\beta e_{3} ;\left[e_{1}, e_{2}, e_{3}\right]=\gamma e_{4}$

$* \operatorname{Der}(\mathfrak{g})=\{\mathrm{D} \in \operatorname{End}(\mathfrak{g})$ such that,

$$
\left.M=\left(\begin{array}{cccc}
0 & a_{12} & a_{13} & a_{14} \\
\alpha a_{12} & 0 & a_{23} & a_{24} \\
-\beta a_{13} & \frac{\beta}{\alpha} a_{23} & 0 & a_{34} \\
\gamma a_{14} & -\frac{\gamma}{\alpha} a_{24} & \frac{\gamma}{\beta} a_{34} & 0
\end{array}\right)\right\}
$$

$* \mathrm{C}(\mathfrak{g})=\{\lambda \mathrm{Id}\}$

* Thus ZDer $(\mathfrak{g})=\{0\}$.

4.1. Classification of $(\alpha, \beta, \gamma, \theta)$-derivations and $(\alpha, \beta, \gamma, \theta)$-quasiderivations. Now we classify, using Theorem 3.35, $(\alpha, \beta, \gamma, \theta)$-derivations in dimension three and dimension four. For this we need to determine the spaces $\mathrm{D}(1, \lambda, 0,0)$ and $\mathrm{QC}(\mathfrak{g})$.

Remark 4.2. If $\lambda=1$, then $\mathrm{D}(1, \lambda, 0,0) \cap \mathrm{QC}(\mathfrak{g})=\mathrm{C}(\mathfrak{g})$. Therefore, in the following computations, we suppose that $\lambda \neq 1$.

Lemma 4.3. Every central derivation of a ternary Lie algebra $\mathfrak{g}$ is an $(\alpha, \beta, \gamma, \theta)$ derivation. 
Proof. Let D be a central derivation of $\mathfrak{g}$, then the image of $\mathfrak{g}$ under $\mathrm{D}$ is a subset of its center. Therefore, for any $x, y, z$ in $\mathfrak{g}$ we have:

$$
[D(x), y, z]=[x, D(y), z]=[x, y, D(z)]=0 .
$$

On the other hand, since $\mathrm{D}\left(\mathfrak{g}^{1}\right)=\{0\}$, so $\mathrm{D}([x, y, z])=0$. Thus $\mathrm{D}$ is an $(\alpha, \beta, \gamma, \theta)$ derivation.

Let $\mathrm{D}$ be an $(\alpha, \beta, \gamma, \theta)$-derivation of $\mathfrak{g}$

(1) If $\operatorname{dim}(\mathfrak{g})=3$,

- $\mathfrak{g}$ is abelian, $\mathrm{D}(\alpha, \beta, \gamma, \theta)=\operatorname{End}(\mathfrak{g})$.

- $\left[e_{1}, e_{2}, e_{3}\right]=e_{1}: \mathrm{D}(\alpha, \beta, \gamma, \theta)=\operatorname{ZDer}(\mathfrak{g})$.

(2) If $\operatorname{dim}(\mathfrak{g})=4$,

- $\left[e_{2}, e_{3}, e_{4}\right]=e_{1}$,

- If $\lambda=0$, then $\mathrm{D}(\alpha, \beta, \gamma, \theta)=\operatorname{ZDer}(\mathfrak{g})$.

- If $\lambda \neq 0$, then the matrix $M$ of $D$ has the form

$$
M=\left(\begin{array}{cccc}
\frac{1}{\lambda} a_{22} & a_{12} & a_{13} & a_{14} \\
0 & a_{22} & a_{23} & a_{24} \\
0 & a_{32} & a_{22} & a_{34} \\
0 & a_{42} & a_{43} & a_{22}
\end{array}\right)
$$

- $\left[e_{1}, e_{2}, e_{3}\right]=e_{1}: D(\alpha, \beta, \gamma, \theta)=\operatorname{ZDer}(\mathfrak{g})$.

- $\left[e_{2}, e_{3}, e_{4}\right]=e_{1} ;\left[e_{1}, e_{3}, e_{4}\right]=a e_{2} ;\left[e_{1}, e_{2}, e_{4}\right]=b e_{3}: D(\alpha, \beta, \gamma, \theta)=$ $\operatorname{ZDer}(\mathfrak{g})$.

- $\left[e_{2}, e_{3}, e_{4}\right]=e_{1} ;\left[e_{1}, e_{3}, e_{4}\right]=a e_{2} ;\left[e_{1}, e_{2}, e_{4}\right]=b e_{3} ;\left[e_{1}, e_{2}, e_{3}\right]=c e_{4}:$ $\mathrm{D}(\alpha, \beta, \gamma, \theta)=\operatorname{ZDer}(\mathfrak{g})$.

\section{References}

[1] J. Arnlind, A. Kitouni, A. Makhlouf and S. Silvestrov, Structure and cohomology of 3-Lie algebras induced by Lie algebras, Algebra, geometry and mathematical physics, 123-144, Springer Proc. Math. Stat., 85, Springer, Heidelberg, 2014.

[2] J. Arnlind, A.Makhlouf and S. Silvestrov, Ternary Hom-Nambu-Lie algebras induced by Hom-Lie algebras, J. Math. Phys., 51(4) (2010), 043515, 11 pp.

[3] J. Arnlind, A. Makhlouf and S. Silvestrov, Construction of $\mathrm{n}$-Lie algebras and n-ary Hom-Nambu-Lie algebras, J. Math. Phys., 52(12) (2011), 123502, 13 pp.

[4] R. Bai, J. Wang and Z. Li, Derivations of the 3-Lie algebra realized by $\mathrm{gl}(\mathrm{n}, \mathbb{C})$, J. Nonlinear Math. Phys., 18(1) (2011), 151-160.

[5] V. T. Filippov, n-Lie algebras, Sibirsk. Mat. Zh., 26(6) (1985), 126-140. 
[6] V. T. Filippov, On $\delta$-derivations of Lie algebras, (Russian) Sibirsk. Mat. Zh., 39(6) (1998), 1409-1422, iv; translation in Siberian Math. J. 39(6) (1998), 1218-1230.

[7] M. E. Gordji, R. Farrokhzad and S. A. Hosseinioun, Ternary $(\sigma, \tau, \xi)-$ derivations on Banach ternary algebras, Int. J. Nonlinear Anal. Appl., 5(1) (2014), 23-35.

[8] M. R. Hestenes, A ternary algebra with applications to matrices and linear transformations, Arch. Rational Mech. Anal., 11 (1962), 138-194.

[9] I. Kaygorodov, On $\delta$-derivations of n-ary algebras, (Russian) Izv. Ross. Akad. Nauk Ser. Mat., 76(6) (2012), 81-94; translation in Izv. Math., 76(6) (2012), 1150-1162.

[10] I. Kaygorodov and Y. Popov, Generalized derivations of (color) n-ary algebras, Linear Multilinear Algebra, 64(6) (2016), 1086-1106.

[11] G. F. Leger and E. M. Luks, Generalized derivations of Lie algebras, J. Algebra, 228(1) (2000), 165-203.

[12] W. G. Lister, A structure theory of Lie triple systems, Trans. Amer. Math. Soc., 72 (1952), 217-242.

[13] P. Novotný and J. Hrivnák, On $(\alpha, \beta, \gamma)$-derivations of Lie algebras and corresponding invariant functions, J. Geom. Phys., 58(2) (2008), 208-217.

[14] J. M. Perez-Izquierdo, Unital algebras, ternary derivations, and local triality, Algebras, representations and applications, Contemp. Math., 483, Amer. Math. Soc., Providence, RI, (2009), 205-220.

[15] Y. Sheng, Representations of hom-Lie algebras, Algebr. Represent. Theory, 15(6) (2012), 1081-1098.

\author{
Amine Ben Abdeljelil and Mohamed Elhamdadi \\ Department of Mathematics \\ University of South Florida \\ FL 33620, Tampa, U.S.A. \\ e-mails: amine@mail.usf.edu (A. B. Abdeljelil) \\ emohamed@math.usf.edu (M. Elhamdadi) \\ Abdenacer Makhlouf (Corresponding Author) \\ Laboratoire de Mathématiques, Informatique et Applications \\ Université de Haute Alsace \\ F-68093, Mulhouse, France \\ e-mail: Abdenacer.Makhlouf@uha.fr
}

